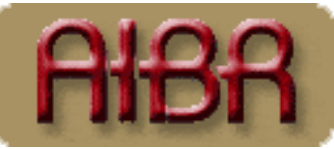

REVISTA DE ANTROPOLOGÍA IBEROAMERICANA

ESTIMADO LECTOR/A:

GRACIAS POR DESCARGAR ESTE ARTÍCULO. EL TEXTO QUE ESTÁ A PUNTO DE CONSULTAR ES DE ACCESO LIBRE Y GRATUITO GRACIAS AL TRABAJO Y LA COLABORACIÓN DESINTERESADA DE UN AMPLIO COLECTIVO DE PROFESIONALES.

USTED PUEDE AYUDARNOS A INCREMENTAR LA CALIDAD Y A MANTENER LA LIBRE DIFUSIÓN DE LOS CONTENIDOS DE ESTA REVISTA A TRAVÉS DE SU INSCRIPCIÓN A LA ASOCIACIÓN AIBR:

http://www.aibr.org/antropologia/aibr/socios.php

La asociación a AIBR tiene un coste mínimo al año, y le proporcionará las siguientes ventajas y privilegios:

1. Recibir en su domicilio la revista impresa, en Europa y América (tres números anuales), así como todas las novedades relativas al funcionamiento de la asociación.

2. Recibir en su domicilio, a precio especial o de forma gratuita, cuantas publicaciones adicionales edite la asociación.

3. Derecho a voto en las asambleas de socios, así como a presentarse como candidato a la elección de su Junta Directiva.

4. Recibir el boletín de socios (tres números anuales), así como la información económica relativa a cuentas anuales de la asociación.

5. Beneficiarse de las reducciones de precio en congresos, cursos, libros y todos aquellos convenios a los que a nivel corporativo AIBR llegue con otras entidades. En este momento, existen los siguientes acuerdos:

o Reducción de un $20 \%$ en el precio de todos los libros publicados por la editorial MELUSINA.

o Reducción de un $20 \%$ en el precio de todos los libros publicados por la editorial SEPHA.

o Reducción de un 30\% en el precio de todos los libros publicados por la editorial GRAN VÍA.

o Derecho a cuota reducida en los congresos trianuales de la FAAEE (España) y a los bianuales de la Sociedad Española de Antropología Aplicada.

o Derecho a cuota reducida en los congresos la IUAES.

6. Promoción gratuita, tanto a través de la revista electrónica como de la revista impresa, de aquellas publicaciones de las que sea autor y que estén registradas con ISBN. La difusión se realiza entre más de 5.000 antropólogos suscritos a la revista.

7. Cuenta de correo electrónico ilimitada de la forma socio@aibr.org, para consultar a través de webmail o cualquier programa externo.

8. Espacio para web personal de la forma http://www.aibr.org/(directorio)/(nombre) y cuenta propia de ftp.

9. Acceso con clave a todos los documentos de la Intranet de socios de AIBR, incluida la consulta a artículos en proceso de evaluación de la revista AIBR.

10. Promoción gratuita a través de la revista (banner rotativo y reseña) de aquellos eventos, congresos, conferencias o cursos en los que usted forme parte del comité organizador.

11. Opción a formar parte como evaluador de los artículos recibidos por la revista.

IMPORTE DE LA CUOTA ANUAL: Hasta diciembre de 2008, la cuota única anual es de 32 (euros). Su validez es de un año a partir del pago de la cuota. Por favor, revise la actualización de cuotas en nuestra web. 


\title{
Espacio crítico
}

\section{P.P LA (ESCASA) INFLUENCIA DE LA www.aibr.org \\ ANTROPOLOGÍA EN LA TOMA DE DECISIONES RELATIVAS A LAS MIGRACIONES EN EL ÁMBITO INTERNACIONAL ${ }^{1}$}

\section{David Martín Castro}

Antropólogo. Actualmente trabaja como Oficial Adjunto de Derechos Humanos en el Alto Comisionado de las Naciones Unidas para los Derechos Human². E-mail: dmartincastro@cps.ucm.es

\begin{abstract}
Resumen
El texto analiza la escasa influencia que tienen los antropólogos tanto en la definición de las agendas como en el contenido de las políticas y normas internacionales en el ámbito de las migraciones. Para ello, utiliza algunos ejemplos de eventos recientes.
\end{abstract}

\section{Palabras claves}

Migraciones, sistema internacional, Naciones Unidas, participación, toma de decisiones

\begin{abstract}
This paper analyzes the limited influence anthropologists have had in the definition of the agenda and in the contents of the policies and international instruments dealing with international migration. In order to do so, some examples from recent events are used.
\end{abstract}

Key words

Migrations, international system, United Nations, participation, decision making process

Recibido: 1 de Agosto de 2007

Aceptado: 23 de Agosto de 2007

\footnotetext{
${ }^{1}$ Este texto parte de una concepción aplicada de la antropología, en la que el antropólogo tiene no sólo la posibilidad sino el deber de participar en la mejora de la situación de las poblaciones con las que trabaja, en coherencia con el trabajo del antropólogo mexicano Eduardo Menéndez.

${ }_{2}^{2}$ El presente artículo no representa la opinión de la Alta Comisionada ni de su oficina, reflejando simplemente la opinión del autor.
} 


\section{La importancia del sistema internacional en la investigación de las migraciones}

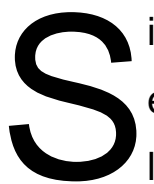

hay una cuestión de carácter internacional que la antropología ha estudiado en los últimos años, esta es la de las migraciones: congresos, libros y cursos sobre la antropología de las migraciones son habituales en los currículos universitarios. El carácter internacional de las migraciones se ve reflejado en que afectan, al menos, a dos ámbitos nacionales diferentes, el país de origen y de destino; si bien normalmente el itinerario migratorio suele incluir varios países de tránsito.

Las migraciones son también uno de los ámbitos donde las legislaciones nacionales adquieren una mayor importancia para la investigación: las leyes de extranjería son básicas para comprender las estrategias migratorias en los países de destino y origen (los "papeles" y la sensación de legalidad o ilegalidad es una cuestión fundamental). Esto ha promovido que muchos antropólogos especializados en este campo se hayan convertido en expertos juristas y asesores legales sobre las leyes de migración.

Los estados receptores de inmigrantes han venido estableciendo unas políticas nacionales cada vez más restrictivas, que han ido aparejadas a un refuerzo de la distinción entre el "nosotros" (nacionales y ciudadanos extranjeros asimilados) y el "ellos" (inmigrantes). En algunos casos, las diferencias legales entre los ciudadanos nacionales y extranjeros son extremas. Tal es el caso de los países del Golfo Pérsico en los que la protección de los derechos humanos, tal y como ha sido denunciado en numerosas ocasiones, es muy deficiente en el caso de los trabajadores inmigrantes ${ }^{3}$.

Simultáneamente a este proceso, se ha ido ensanchando la división entre aquellos inmigrantes que entran legalmente y que, con el tiempo, van adquiriendo cada vez más características del "nosotros", y aquellos que no poseen un estatus de residencia legal y que se ven condenados a ser "ellos" indefinidamente o, por lo menos, por un largo periodo de tiempo.

\footnotetext{
${ }^{3}$ Dos de los órganos internacionales que más han estudiado esta situación han sido el Programa para Asia del Fondo de de Desarrollo de Acciones Unidas para la Mujer (UNIFEM) y los informes del Relator Especial de la Comisión de Derechos Humanos (y asumido por el Consejo de Derechos Humanos) sobre los derechos humanos de los migrantes.
} 
La existencia de estas políticas restrictivas ha creado no pocos conflictos internacionales, que van desde la queja de México por la construcción de un muro de separación en la frontera que comparte con Estados Unidos (al tiempo que aplaude la posible legalización de 12 millones de inmigrantes en EEUU), a que se condicione la ayuda al desarrollo a la aceptación de convenios de repatriación de inmigrantes, política que han venido llevando a cabo varios países europeos con países del África Subsahariana.

A pesar de este carácter internacional, en pocas ocasiones los estados han permitido que estos asuntos se decidan fuera de sus relaciones bilaterales, reclamando para sí mismos el respeto absoluto a su soberanía nacional.

La coordinación de las políticas nacionales en el ámbito de las migraciones y el establecimiento de relaciones fluidas intergubernamentales se han mostrado muy útiles para evitar el surgimiento de conflictos en el ámbito internacional. Por ello, se ha realizado en los últimos diez años un importante número de esfuerzos en la coordinación de las políticas y los flujos migratorios, y que reflejan la importancia que se ha dado a esta cuestión en el ámbito internacional. Como protagonistas de estos esfuerzos, destacan los siguientes organismos:

- La Organización Internacional para las Migraciones: tiene, entre otros, los mandatos de producir conocimiento (recopilando legislaciones nacionales, regionales e internacionales y ofrecerlos al público) y de fomentar acuerdos entre los estados miembros sobre las migraciones.

- La Organización Internacional del Trabajo (OIT): ha llevado a cabo una política muy activa para la protección de los derechos de los trabajadores migrantes, entre los que destaca la adopción de los convenios 97 y 143 sobre trabajadores inmigrantes ${ }^{4}$, si bien entre ambos convenios apenas se supera la cincuentena de ratificaciones ${ }^{5}$. Además, la OIT lleva a cabo otras actividades y proyectos de promoción y protección de los derechos laborales de los inmigrantes.

- El sistema de derechos humanos de las Naciones Unidas: ha sido en este ámbito donde se han analizado de forma más exhaustiva los derechos de los migrantes. En 1990, se adoptó la Convención internacional sobre la protección de los derechos de todos los trabajadores migratorios y de sus familiares, por la que se establece un comité responsable de velar por su

\footnotetext{
${ }^{4}$ Dichos convenios están disponibles en http://www.ilo.org/ilolex/spanish/convdisp1.htm

${ }^{5}$ A pesar del relativamente escaso número de ratificaciones recibidas, estos convenios muestran la opinión internacional sobre lo que debería hacerse para proteger sus derechos.
} 
cumplimiento (Comité de los Trabajadores Migrantes -CMW) ${ }^{6}$. Asimismo, la extinta Comisión de Derechos Humanos (reemplazada por el Consejo de Derechos Humanos en 2006) estableció, en 1999, la Relatoría Especial sobre los derechos humanos de los migrantes. Esta relatoría tiene el mandato de analizar y vigilar la situación de los derechos humanos de los migrantes. En la actualidad, la relatoría está ocupada por J.A. Bustamante, antropólogo y sociólogo mexicano ${ }^{7}$.

Junto a estos instrumentos y mecanismos del ámbito internacional, se han abierto varios procesos globales en los que se vienen debatiendo políticas relativas a las migraciones. Entre de estos procesos destacan:

- Grupo Global sobre las Migraciones (Global Migration Group): En él se reúnen las diferentes agencias del sistema de las Naciones Unidas para coordinar sus políticas, actividades y acciones en este ámbito ${ }^{8}$.

- Comisión Global sobre Migraciones Internacionales: fue recientemente lanzada por el Secretario General con el fin de proporcionar un marco coherente para la actuación internacional en este ámbito ${ }^{9}$.

- Ligado con el anterior, el Foro Global de las Migraciones y el Desarrollo: recientemente promovido por el Secretario General y estados miembros, es un foro de debate y concertación sobre políticas migratorias. El último Foro tuvo lugar en Bélgica en julio de $2007^{10}$. Existe una sección de sociedad civil y otra de estados.

En estas reuniones internacionales se observa cada vez más una ruptura entre las posiciones de los países emisores y las de los receptores de migrantes. Uno de los ejemplos más gráficos es la lista de ratificaciones de la Convención internacional sobre la protección de los derechos de todos los trabajadores migratorios y de sus familiares. En sus 93 artículos, la Convención reconoce un amplio elenco de derechos a los trabajadores migratorios y sus familiares, basados

${ }^{6}$ El mandato y los documentos producidos por el Comité se encuentran disponibles en http://www.ohchr.org/english/bodies/cmw/index.htm Además, se publicó recientemente la revisión del documento "La convención internacional de los trabajadores migrantes y su comité. Folleto informativo número 24 de la OACNUDH.

7 Para más información sobre la Relatoría ver http://www.ohchr.org/english/issues/migration/rapporteur/

${ }^{8}$ Ver http://www.ohchr.org/english/bodies/cmw/GMG.htm . En dicha página puede encontrarse una breve referencia sobre el trabajo que las diferentes agencias de Naciones Unidas llevan a cabo en relación con las migraciones.

${ }^{9}$ Ver http://www.gcim.org/es/

${ }^{10}$ Ver http://www.gfmd-fmmd.org/lang.html 
principalmente en el principio básico de derechos humanos de no discriminación ${ }^{11}$. Debido a su carácter obligatorio y al amplio reconocimiento de derechos, los países receptores de inmigrantes, especialmente europeos, no la han ratificado y aprovechan los foros donde se analizan cuestiones ligadas (como por ejemplo el Consejo de Derechos Humanos) para reafirmar su falta de disposición a hacerlo al considerar que no es un instrumento válido para garantizar los derechos de los migrantes $^{12}$. Sin embargo, una parte muy importante de los derechos reconocidos están implícitos en otros instrumentos de protección de los derechos humanos, especialmente la Convención Internacional sobre la Eliminación de todas las Formas de Discriminación Racial, que ya han ratificado en el pasado.

Además de estos foros, existe una enorme producción de documentos oficiales de Naciones Unidas, producidas por la CEPAL ${ }^{13}$, la Oficina del Alto Comisionado para los Derechos Humanos ${ }^{14}$, DESA (Departamento de Asuntos Económicos y Sociales del Secretariado de la ONU $)^{15}$, la Organización Internacional para las Migraciones que reflejan el trabajo que lleva a cabo el sistema de las Naciones Unidas en este ámbito. En todos ellos, se observa que los derechos humanos y la necesidad de racionalizar las barreras migratorias van ganando terreno.

Si bien estas reuniones, documentos e instituciones pueden parecer muy alejadas de las preocupaciones y de los problemas de los que se ocupan la mayor parte de las investigaciones antropológicas en este ámbito, lo cierto es que tienen una gran influencia, por cuanto afectan indudablemente en el contexto en el que se producen las migraciones, ya que son normalmente considerados "textos de autoridad", en los que algunos estados llegan a basar o justificar políticas nacionales y regionales.

No obstante, y más allá del contenido de los documentos producidos, la importancia de estos foros y organizaciones también radica en el hecho de que son lugares de diálogo y concertación de acciones, tanto entre los estados como con la sociedad civil. Las reuniones paralelas entre estados, entre estados y sociedad civil, y las

${ }^{11}$ El principio de no discriminación ya venía recogido en la Declaración Universal de Derechos Humanos (1948).

${ }^{12}$ Ejemplos de esta opinión pueden encontrarse en las actas del Consejo de Derechos Humanos tanto en las fechas de su aprobación como en las respuestas de los estados en el diálogo anual con el Relator Especial sobre los derechos humanos de los migrantes en el Consejo de Derechos Humanos. Más información en http://www.ohchr.org/spanish/bodies/hrcouncil/index.htm. Sobre la posición de los países de la UE en este ámbito, ver MacDonald y Cholewinski (2007).

${ }^{13}$ La más conocida es probablemente CEPAL (2006).

14 Principalmente a través de los informes del Relator Especial, accesibles en http://ap.ohchr.org/documents/dpage e.aspx?m=97

${ }^{15}$ Uno de los documentos de DESA más interesantes es DESA 2005. 
presentaciones de políticas específicas en los plenarios, ayudan a los policy makers a conocer las acciones llevadas a cabo en otros países y a elaborar sus propias políticas nacionales y a tomar medidas coordinadas de acción. La replica de políticas migratorias y de las "buenas" prácticas surgen en muchos casos de estas reuniones paralelas.

\section{La influencia de la antropología de las migraciones en el ámbito internacional}

Ante este panorama, cabe preguntarse cuál ha sido hasta el momento el efecto que los estudios antropológicos de las migraciones han tenido en estos foros y documentos internacionales. La verdad, es que el panorama es bastante desolador y se observa que en muy pocos casos puede decirse que detrás de las políticas llevadas a cabo internacionalmente hay antropólogos.

Los participantes en las reuniones internacionales sobre las migraciones son en su mayoría economistas y juristas, siendo la participación de antropólogos marginal (más allá del papel destacado que puedan haber tenido algunos antropólogos específicos). No se trata tanto de qué expertos participan sino de la formación que tienen los funcionarios que definen las prioridades en estos informes. Esta situación es diferente en dos ámbitos ligados a las migraciones. Estos ámbitos son relativamente marginales dentro del proceso y formas de toma de decisiones, y están muy ligados con los preconceptos del papel que juega la antropología dentro de las ciencias sociales.

Esta situación de marginación se acentúa en los dos grandes momentos del proceso de toma de decisiones: la elaboración de la agenda y la definición de los documentos que sirven de base para la discusión y la toma de decisiones. En ambos casos parece ser que nuestra disciplina tiene poca influencia.

La definición de la agenda es, sin lugar a dudas, el punto central de todo proceso de negociación y en la definición de la misma no se consideran relevantes los aspectos donde la aportación de la antropología podría ser mayor. En este sentido, si se analizan las agendas de los foros internacionales anteriormente mencionados, se observa que las discusiones internacionales se centran casi siempre en sus aspectos económicos o legales (lo que está ligado a la formación que poseen los funcionarios que trabajan en estos ámbitos). Este hecho conlleva 
que la definición de los problemas en el ámbito internacional se aleje de las principales cuestiones analizadas por la antropología o allí donde sus aportaciones podrían ser más valoradas.

El ejemplo más reciente es el informe del Día de la Sociedad Civil del Foro Global de las Migraciones y el Desarrollo, que tuvo lugar el 9 de julio de 2007, y que podría parecer el ámbito internacional de más fácil acceso para los antropólogos. Dichos resultados, que tienen la forma de conclusiones y recomendaciones, se basan en un análisis económico y legal de las migraciones. El formato de la discusión, muestra el tipo de análisis del debate:

- Desarrollo de capital humano y movilidad laboral: maximización de las oportunidades y minimización de los riegos

- Remesas y otros recursos de la diáspora: aumentar su valor neto y el valor de su desarrollo

- Incremento de la coherencia política e institucional y promoción de alianzas

La lectura completa del documento de conclusiones y recomendaciones muestra que la aportación de la antropología en las discusiones fue, cuanto menos, limitado $^{16}$. Pero ¿cómo podría ser de otro modo? La elección de la temática responde a una agenda de trabajo y de definición de prioridades donde la antropología es accesoria.

No obstante, si en vez de centrarnos en un documento del segmento de la sociedad civil, donde podría parecer que los antropólogos podrían haber tenido una mayor participación, nos centramos en el documento final del segmento de los estados, el panorama es aún más desolador. De todas formas, ¿cómo van a discutir los Estados desde una perspectiva o con una base mínimamente antropológica si la sociedad civil, en su reunión previa, no ha considerado importante estos asuntos?

Un segundo aspecto a tener en cuenta, mas allá de la definición de la agenda internacional, son los informes y estudios en los que se basa la definición internacional de la situación de las migraciones. El informe del Secretario General de la ONU para la Reunión de Alto Nivel "migraciones y desarrollo" (documento ONU A/60/871), que tuvo lugar a finales de 2006, representa la visión que sobre las migraciones tiene la secretaría del sistema de Naciones Unidas. A diferencia del foro

\footnotetext{
${ }^{16}$ Para más información, ver http://www.gfmd-civil-society.org/index.html. El informe se encuentra en http://smooz.gfmd-civil-society.org/gfmd/files/Final CSD.pdf
} 
anteriormente mencionado, que tenía como objetivo el establecimiento de recomendaciones de políticas, el informe del Secretario General es un estudio sobre la situación y la problemática de las migraciones internacionales. En asuntos tales como las comunidades transnacionales, el regreso de los emigrantes, podría ser esperable que la aportación de la antropología fuera mayor. A modo de ejemplo, en comunidades transnacionales se señala:

59. Conscientes de que sus ciudadanos que trabajan en el extranjero pueden constituir activos para el desarrollo, los gobiernos están reforzando sus vínculos con ellos. Las remesas colectivas de las asociaciones de migrantes que financian proyectos de desarrollo en pequeña escala ya están mejorando la vida en las comunidades de origen, a menudo con el apoyo de las autoridades locales y nacionales. En tanto que consumidores, los migrantes contribuyen a la expansión del comercio, el turismo y las telecomunicaciones en sus países de origen y destino. Además, a menudo se convierten en empresarios, ya sea en los países de destino o una vez que regresan a sus comunidades originarias, creando negocios que pueden generar riqueza y empleo.

60. Los migrantes promueven además la inversión extranjera en sus países de origen con sus propias inversiones y también reduciendo barreras comerciales basadas en la reputación del país y aprovechando sus contactos en el mundo de los negocios. Las redes que unen a los trabajadores con conocimientos científicos y técnicos del país de origen con sus homólogos migrantes en el extranjero permiten la transferencia de conocimientos especializados de tipo productivo y tecnológico.

Es legítimo plantearse cómo es posible que en un aspecto que ha sido tan estudiado por la antropología, los y las antropólogos no hayan tenido ningún tipo de influencia en la redacción de estos párrafos, ni en ningún otro de sus más de noventa páginas.

Una de las explicaciones se encuentra en su bibliografía. En las más de 100 referencias recogidas, se observa que los estudios académicos escritos por antropólogos tienen una escasa representación, por no decir que es casi inexistente, no mencionándose siquiera una referencia a J.A. Bustamante, actual Relator Especial sobre los derechos de los migrantes.

Sin embargo, los antropólogos sí vienen participando en dos de los ámbitos internacionales en los que se toman decisiones ligadas a las migraciones. En ambos casos, y siguiendo el conocido artículo de Precht (1980), los nichos ocupados por la antropología son los tradicionales que las ciencias sociales han otorgado a nuestra ciencia.

Por un lado, si hay un ámbito internacional en el que la participación de antropólogos es relativamente importante, éste es el de las migraciones de los 
pueblos indígenas ${ }^{17}$. En los dos seminarios principales donde se han analizado estas cuestiones, han sido varios los antropólogos que participaron en las discusiones. Es necesario resaltar que, en el ámbito internacional, los pueblos indígenas, mucho más que las minorías o cualquier otro grupo humano, se han constituido en los "otros" ${ }^{\text {". }}$. Sin embargo, aún reconociendo la gran importancia que ello tiene, el ámbito de las migraciones indígenas es un nicho alejado de los centros de decisión o de la parte "más dura" del debate internacional sobre las migraciones. En las reuniones de política general a nivel global, rara vez se constituyen en algo más que un punto en la agenda.

Junto a este ámbito, destaca el papel del actual Relator Especial sobre los derechos humanos de los migrantes, el antropólogo J.A. Bustamante. El Relator Especial tiene como mandato tanto realizar estudios sobre la situación de los derechos humanos de los migrantes (a través de informes temáticos y visitas a países) como mantener comunicaciones con los estados sobre posibles violaciones de sus derechos humanos en los ámbitos nacionales ${ }^{19}$.

Los Relatores Especiales son expertos independientes que, nombrados por el Consejo de Derechos Humanos, representan un valor moral y de salvaguarda en la protección de los derechos. En este sentido, no representan a la organización sino que simplemente dan sus visiones particulares como expertos. La consideración de sus textos depende mucho de la persona que ocupa el cargo.

Los informes del Relator Especial sobre los derechos humanos de los migrantes tienen una buena acogida internacional y son textos de gran relevancia y una importante fuente de información. Especialmente relevante son sus informes anuale ${ }^{20}$. Entre ellos, destaca el informe de 2007, que se basa en la información proporcionada por los estados a un cuestionario sobre las repercusiones que han tenido ciertas leyes y medidas administrativas sobre los migrantes.

\footnotetext{
${ }^{17}$ Hasta la fecha, se han organizado dos seminario internacionales específicamente centrado en las migraciones indígenas, ambos en el ámbito del Foro Permanente para las Cuestiones Indígenas. El primero de ellos se llevó a cabo en Ginebra, del 6 al 7 de abril de 2006 (Informe del seminario, Pueblos Indígenas y Migraciones: Retos y Oportunidades accesible en http://www.un.org/esa/socdev/unpfii/documents/5session crp5 migration.doc) y el segundo en Santiago de Chile, del 27 al 29 de marzo de 2007 (Informe del seminario, Pueblos Indígenas Urbanos y Migraciones accesible en http://www.un.org/esa/socdev/unpfii/documents/6session crp8 es.doc

${ }^{18}$ Esta cuestión se observa principalmente por el reconocimiento de derechos particulares a los pueblos indígenas.

19 En su informe anual de 2006 (E/CN.4/2006/73) presentado ante la Comisión de Derechos Humanos en su $62^{\mathrm{a}}$ y última sesión analiza el mandato del propio relator.

${ }^{20}$ Accesibles en http://ap.ohchr.org/documents/dpage e.aspx?m=97 
Sin embargo y a pesar del gran valor moral y relevancia de los Relatores Especiales, rara vez sus recomendaciones y conclusiones (quitando algunas honrosas excepciones) consiguen entrar en las agendas políticas nacionales, si bien en algunos casos si se consigue entablar un diálogo constructivo. A modo de ejemplo, la anterior Relatora, la costarricense Gabriela Rodríguez Pizarro, mantuvo un interesante diálogo con el Gobierno de España en seguimiento a la visita que realizó en 2003 a España. Allí, tanto la Relatora como el Gobierno de España debatieron las conclusiones y recomendaciones recogidas en el informe ${ }^{21}$.

\section{Conclusiones}

La influencia de la antropología en el ámbito internacional de toma de decisiones sobre las migraciones es, cuanto menos, muy limitada. En ocasiones, puede considerarse que esto se debe a que en estos ámbitos se discuten cuestiones ligadas a la elaboración de políticas o asuntos económicos en los que la antropología tiene dificultades para establecer su agenda. Pero en muchas otras, tales como la definición de la situación específica y los problemas a tratar, se observa que los antropólogos (y no hablamos ni siquiera del ámbito académico) no han conseguido influir, ni sus estudios alcanzar a aquellos que toman las decisiones. Es importante señalar que muchos de los estudios antropológicos tienen un carácter local que no parece suficiente para explicar el silencio existente. En ocasiones, pareciera que muchos antropólogos han decidido centrarse en los aspectos locales y en situaciones concretas, sin que tomen en consideración la importancia que tiene que sus trabajos e influencia alcancen los ámbitos de decisión internacionales en los que se establecen el contexto en el que se mueven sus investigaciones.

Al mismo tiempo, es en aquellos ámbitos relativamente marginales, como las migraciones de los pueblos indígenas, donde la antropología parece mostrar la imagen que nuestra disciplina tiene en el ámbito internacional. Aún reconociendo la centralidad de dicha cuestión, ésta no se encuentra en el centro de las discusiones internacionales sobre las migraciones internacionales.

\footnotetext{
${ }^{21}$ Ver los documentos E/CN.4/2004/76/Add.2 y E/CN.4/2004/G/17. Accesibles en la página mencionada en la nota anterior.
} 
Mientras no se participe en la definición de las agendas y no se consiga que las publicaciones y estudios llevados a cabo por antropólogos lleguen a influir en los cuerpos de expertos que elaboran las políticas internacionales relativas a las migraciones, será muy difícil que, más allá de los ámbitos locales y asistenciales, la antropología consiga realmente modificar y elaborar políticas de migración que atiendan las necesidades de estas poblaciones. Asimismo, sólo con esta participación podría conseguirse modificar la imagen que de la antropología existe en el ámbito internacional.

Lograrlo no debería parecer tan complicado. Se ha mostrado la utilidad e importancia de nuestro trabajo en los ámbitos locales. A partir de ello, se trataría de ir participando, poco a poco, en los ámbitos de la sociedad civil en los que se preparan las reuniones internacionales, incluyendo la solicitud de estatus consultivo de ONGs ante el ECOSOC para las organizaciones de antropólogos más activas. Junto a este proceso, sería necesario que cada vez más antropólogos mostraran su interés por participar en este ámbito y cada vez hubiera más funcionarios internacionales con una formación antropológica. Ya han pasado muchos años desde que se constituyó nuestra disciplina, quizás ahora sea el momento adecuado para dar el paso.

\section{Referencias}

Asamblea General de la ONU (1948), Declaración universal de Derechos Humanos. En http://www.unhchr.ch/udhr/lang/spn.htm

CEPAL (2006), Migraciones internacionales, derechos humanos y desarrollo. Santiago de Chile: CEPAL

Comisión de Derechos Humanos (2004), Informe presentado por la Relatora Especial, Sra. Gabriela Rodríguez Pizarro, de conformidad con la resolución 2003/46 de la Comisión de Derechos Humanos E/CN.4/2004/76/Add.2.

Comisión de Derechos Humanos (2005), Informe del Relator Especial sobre los derechos humanos de los migrantes, Jorge Bustamante E/CN.4/2006/73

DESA (2005), Women and International Migration. NY: ONU.

Foro Permanente para las Cuestiones Indígenas (2006), Pueblos Indígenas y Migraciones: Retos y Oportunidades. Actas del Seminario de Expertos sobre Migraciones Indígenas (Abril Ginebra).En http://www.un.org/esa/socdev/unpfii/documents/5session crp5 migration.doc 
Global Forum on Migration and Development (2007), Report of the Civil Society Day of the Global Forum on Migration and Development. En http://smooz.gfmd-civilsociety.org/gfmd/files/Final CSD.pdf

Gobierno de España (2004) Nota verbal de fecha 29 de enero de 2004 dirigida a la Oficina del Alto Comisionado de las Naciones Unidas para los Derechos Humanos por la Misión Permanente de España ante la Oficina de las Naciones Unidas en Ginebra E/CN.4/2004/G/17

MacDonald, E. y Cholewinski, R (2007), The migrant workers convention in Europe. Obstacles to the Ratification of the International Convention on the Protection of the Rights of All Migrant Workers and Members of their Families: EU/EEA Perspectives. Paris: UNESCO. Accesible en http://unesdoc.unesco.org/images/0015/001525/152537E.pdf

ONU (1990), Convención internacional sobre la protección de los derechos de todos los trabajadores migratorios y de sus familiares. En http://www.ohchr.org/spanish/law/cmw.htm

Secretario General de la ONU (2006), Migración internacional y desarrollo. Informe del Secretario General A/60/871

UN Habitat (2007), Informe del seminario Pueblos Indígenas Urbanos y Migraciones. Documento presentado en la sexta sesión del Foro Permanente para las Cuestiones Indígenas (Nueva York). En http://www.un.org/esa/socdev/unpfii/documents/6session crp8 es.doc 